\title{
Applying Idea Management System (IMS) Approach to Design and Implement a collaborative Environment in Public Service related open Innovation Processes
}

\author{
Marco Alessi ${ }^{1}$, Alessio Camillò ${ }^{2}$, Valentina Chetta ${ }^{2}$, Enza Giangreco ${ }^{2}$, \\ Mona Soufivand ${ }^{1}$ and Davide Storelli ${ }^{2}$ \\ ${ }^{1}$ Engineering ingegneria informatica SPA, R\&D Department, Palermo, Italy \\ ${ }^{2}$ Engineering ingegneria informatica SPA, R\&D Department, Lecce, Italy \\ marco.alessi@eng.it, alessio.camillo@eng.it, valentina.chetta@eng.it, enza.giangreco@eng.it, \\ m.soufivand@gmail.com, davide.storelli@eng.it
}

\begin{abstract}
Novel ideas are the key ingredients for innovation processes, and Idea Management System (IMS) plays a prominent role in managing captured ideas from external stakeholders and internal actors within an Open Innovation process. By considering a specific case study, Lecce-Italy, we have designed and implemented a collaborative environment, which provides an ideal platform for government, citizens, etc. to share ideas and co-create the value of innovative public services in Lecce. In this study the application of IMS with six main steps, including: idea generation, idea improvement, idea selection, refinement, idea implementation, and monitoring, shows that this, remarkably, helps service providers to exploit the intellectual capital and initiatives of the regional stakeholders and citizens and assist service providers to stay in line with the needs of society. Moreover, we have developed two support tools to foster collaboration and transparency: sentiment analysis tool and gamification application.

Keywords: Idea Management System (IMS), value co-creation, Open Innovation, e-government, sentiment analysis, gamification.
\end{abstract}

\section{Introduction}

Value co-creation is an approach to create innovative services. Co-creation is the process by which products, services, and experiences are developed jointly by companies, their stakeholders, and final customers, opening up a new world of value [1]. A new way of conceiving the provision of public services in a mutual relationship among service providers, professionals, service users, and citizens, making these services much more effective, efficient, and far more sustainable [2].

Progress in technologies such as Web 2.0 phenomenon [3], offers the ideal platform for service providers, users and other actors to communicate and interact with each other for exchanging ideas and opinions, which are necessary (but not sufficient) to foster the process of value co-creation.

Great ideas are the key parameters of innovation process for organizations and communities. The ideas flowing without a proper managing mechanism to evaluate, categorize, and prioritize them, would not assist innovation process. As stated by Geoff Mulgan [4], "Innovation is often given complex definitions," but he prefers the simple one: "new ideas that work" [4]. 
Reviewing related literature shows the importance of ideas in the innovation processes. As an example, the European Foundation for Quality Management (EFQM) ${ }^{1}$ defines innovation as "the practical translation of ideas into new or improved products, services, processes, systems or social interactions".

Ven and Poole (1990) [5] argue that "invention is the creation of a new idea, but innovation is more encompassing and includes the process of developing and implementing a new idea. The development of innovation is not a linear process (a pipeline of sequential processes), but it needs a systemic approach". Therefore, innovation starts with 'management of ideas' [6].

The formal process such as Idea Management System (IMS) to structure the aforementioned stages including: capture, filter, evaluation, and implementation of the best ideas, seems essential. Lack of this system may cause superfluous innovation efforts [7].

The complex interactions among many individuals, organizations, and their operating environment is an open innovation process [8], [6]. Chesbrough defines open innovation as: "the use of purposive inflows and outflows of knowledge to accelerate internal innovation, and expand the markets for external use of innovation, respectively. Open innovation is a paradigm assuming that firms can and should use external ideas as well as internal ideas, and internal and external paths to market, as the firms look to advance their technology" [9]. It is in order to drive structural changes far beyond the scope of what any organization could do alone. On the other hand, open innovation was first defined by Henry Chesbrough as opening up the process of innovation to external stakeholders. He expresses that "due to the global completion which leads to the shorter innovation cycles, companies should tap expertise and creativity outside the organization working imperative" [10], [11].

The Open Government (OG) concept, which emphasizes on including citizens and society as well as administration members within governmental processes, is a translation of open innovation in governmental processes. OG seeks to engage citizens in order to increase efficiency within political/organizational decision process leading to society's satisfaction [12].

In this study, we propose a conceptual framework describing the idea of life-cycle and the tools enabling collaboration between citizens and Public Administration, with particular focus on Idea Management System and its role in each step. Moreover, we propose a sentiment analysis within the context of the public administration, with the purpose to provide reliable estimates and analysis of what citizens think about the institutions, the efficiency of services and infrastructures, and the degree of satisfaction about special events.

The proposed IMS is not only a tool for collecting ideas, but it is a collaborative tool for decision support and a tool to support a democratic society where people participate proactively.

To meet the needs of public administration dictated by the new standards of the Open Government, IMS proposed in this paper, reached the following results: (1) Transparency in the decisions made by Public Administration; (2) Bottom up approach; (3) Citizen's active participation; and (4) Development of services more responsive to the users' needs.

The paper is structured as follows. The problem description is given in the next section. Conceptual framework is discussed in Section 3. Lecce 2019 - IMS case study is described in Section 4. Related works are discussed in Section 5. Section 6 concludes the paper.

\section{Problem Description}

According to Edelmann, governments are aware of the significance of citizens' engagement in decision-making processes by integrating their potential in innovation process and acquiring better outcome [12], which reflects a paradigm shift in public administration. However, as stated by Collm and Schedler [13], innovation process in public sector, up to now, has occurred in closed-off processes mainly handled by internal public administration and sometimes with the consultancies support [13].

\footnotetext{
${ }^{1}$ http://www.efqm.org/
} 
Public administration has understood the need to encourage stakeholders and citizens to participate, nevertheless, it still has not found its role in the virtual atmosphere [12].

The ubiquitous presence of ICT together with the recent willingness of citizens to participate and contribute online can enable government agencies to restructure their interaction with citizens in order to achieve better collaboration results [14].

IMS has been successfully implemented in private sector with the purpose of identifying the real demands in order to generate services and products based on them [7]. However, the current discussion on open innovation has hardly touched upon the public sector. For example, Brunswicker has investigated the possibility to apply crowdsourcing platform in the governmental context. These studies showed that design principles derived from open innovation projects in the corporate world may not be directly applied in the governmental context: they need to be adjusted and integrated [15].

Recently, Twitter, one of the most popular micro-blogging tools, has gained significant popularity among the social network services. Micro-blogging is an innovative form of communication, in which users can express, in short posts, their feelings or opinions about a variety of subjects or describe their current status.

Much of the research is particularly interested in the Sentiment Analysis of Twitter messages and tweets. The brevity of these texts (the tweets cannot be longer than 140 characters) and the informal nature of social media, have involved the use of slang, abbreviations, new words, URLs, etc. These factors together with the frequent misspellings and improper punctuation make more complex the understanding of the opinions and feelings of the people.

Despite this, recently, numerous studies have focused on natural language processing of Twitter messages [16], [17], [18], [19], leading to useful information in various fields, such as: brand evaluation [16], public health [20], natural disasters management [21], social behaviors [22], movie market [23], and political sphere [24].

In this paper we present the Idea Management System ${ }^{2}$ developed in the Puglia@service ${ }^{3}$ project, supporting the co-creation activities in the initiative for Lecce candidacy as European Capital of Culture $2019^{4}$.

\section{Conceptual Framework}

The proposed idea life-cycle is characterized by the following six steps (Figure 1):

- Idea Generation

- Idea Improvement

- Idea Selection

- Refinement

- Implementation

- Execution and Monitoring

Each step is carried out in collaboration with citizens or between citizens and public administration; it is characterized by tools that allow the responsible of each step to perform the functions in a collaborative way.

IMS, starting from designed process in BPM, gives users the opportunity to create a social network where they can share, vote, and promote ideas. This environment is designed around local government and citizen needs and provides an engagement approach more efficient and effective than the usual BPM interfaces.

\footnotetext{
${ }^{2}$ http://www.2019idee.eu/

${ }^{3}$ Objective of the project is to start creating a new service culture over the regional territory guiding the transition of Puglia region toward the "smart territory" paradigm. http://www.pugliasmartlab.it

${ }^{4} \mathrm{http}$ //ec.europa.eu/culture/tools/actions/capitals-culture_en.htm
} 


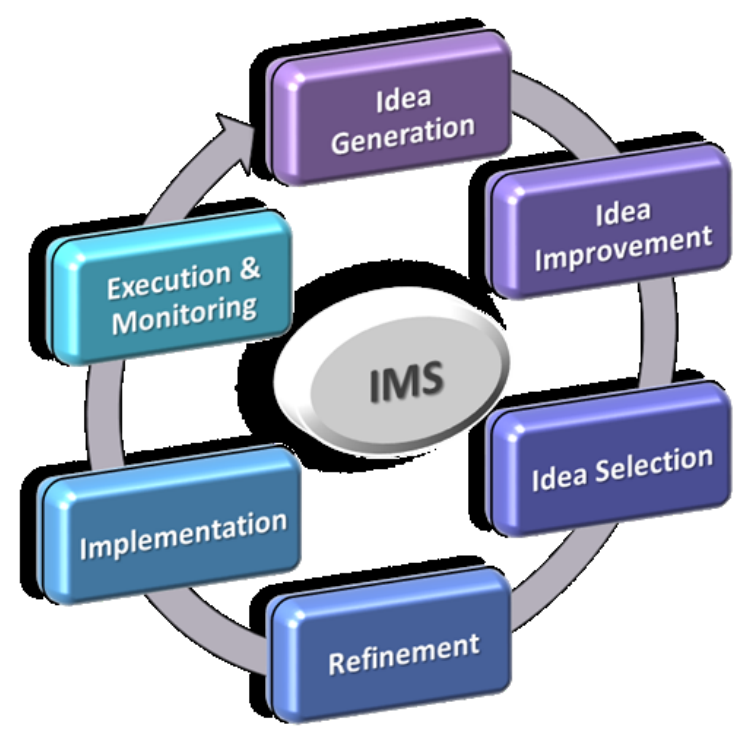

Figure 1. Main steps of idea life-cycle

Idea Generation. This is the phase of ideas input from users. It can take place according to two techniques: Push (the ideas about particular topics are required from public administrator) and Pull (citizen can suggest ideas to Public Administration). The actors involved are Public Administration and citizens. The importance of this phase is the free expressions of citizens able to generate ideas of common and public utility, and encouraging service of co-creation and the participation in the "res nostra".

Idea Management System supports the idea collection, contest creation, and allows the idea sharing on the most important social networks in order to encourage discussion and the promotion of the IMS. Tags and categorization of ideas allow simplifying the idea organization and research.

Idea Improvement. This is the collaboration phase and collective development of ideas. Once generated, ideas are shared and improved thanks to the continuous collaboration between users, who may, through the Idea Management System, contribute to the enrichment of ideas with comments, pictures, links, etc. In this way, from one or more initial ideas a process of cocreation, socialization, and exchange of experience and knowledge is triggered.

Ideas are made available to the whole community that collaborates to transform them into a structured project. Therefore the community, properly supported, can improve ideas, exploiting know-how and multiple perspectives emerging from the system. To encourage the engagement of the citizen and to create participatory behavior, gamification tools were developed.

Idea Selection. This phase supports the evaluation, selection, and ranking of Ideas. Idea Management System allows voting for the ideas that leads to a ranking. This ranking points out ideas with greater priority or the ones considered by users to be better than others. The indicators used for the evaluation are, for example: the number of threads or the vitality index that expresses how the idea remains active over time. In addition, it is possible to make even an indirect analysis of ideas through sentiment analysis that allows identifying the issues particularly important for the citizen/user. The output of this phase is the selection of ideas to be analyzed in detail by studying the sustainability. Charts show the most popular ideas and suggest the most active members of the community. In this way, the ideas most read, commented, or appreciated, emerge and are highlighted more than others. The actors involved are both citizens and the Public Administration (PA). In addition to Idea Management System, a tool of sentiment analysis and a dashboard that shows (both to the PA and citizens) intuitively the data collected have been implemented. Figure 2 illustrates the sentiment analysis dashboard useful to select the most popular ideas. 


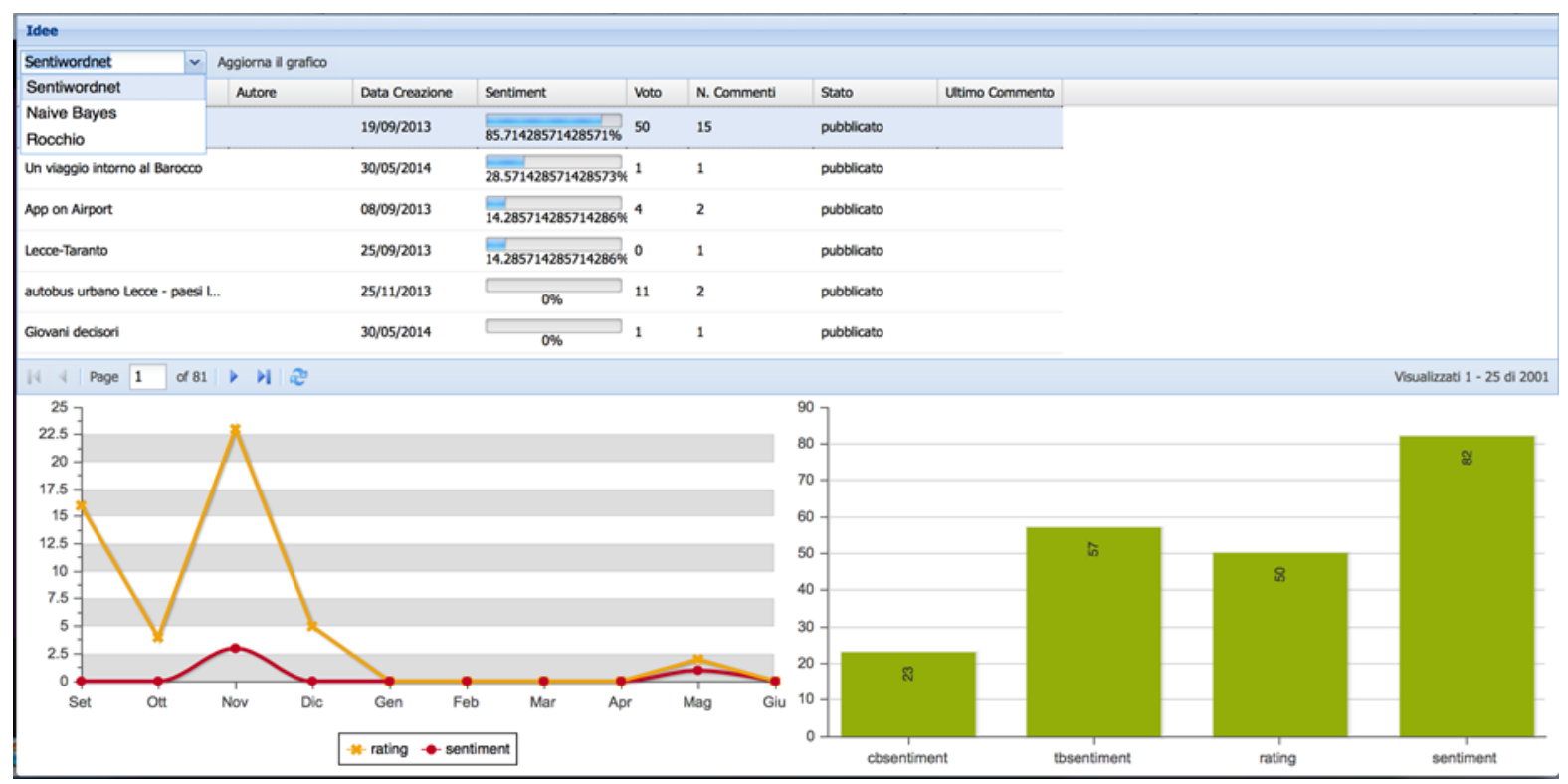

Figure 2. Sentiment analysis dashboard

Refinement. In this phase the selected ideas are refined thanks to the involvement of expert users (citizens or employees of PA) able to describe in detail all the steps and evolutionary processes that accompany the same idea. The expert group is formed through social office tools, by comparing the roles specified by the author of the idea and the co-authors, as necessary for the execution and implementation of the idea itself. The skills available in the profile of users are entered during Idea Management System registration. To assess the social and economic sustainability of the idea, the methodology [25] based on the Value Network Analysis [26] supported by simulation tools is developed. Cooperation and communication between the two actors and the Idea Management System become essential to support this functionality. The output of this phase is the transformation of the idea into a sustainable product/service in technological, economic, and social terms. So, it is important to identify the role of the user, through the tools of the social office.

Implementation. The actors involved in this phase are both the PA and citizens, experts and not experts.

When the ideas require the development of an application and/or an information service, IMS provides a collaborative tool, allowing the user to report the needs useful to develop a service. This notification can supply documentation and models created using the tool. The technologist will try to implement the new service by the integration of existing applications in the marketplace. Where it is necessary to develop a new application that is not in the marketplace, the tool allows the user to report these needs to technologist (in this case the notification shall be accompanied by documentation and models that clarify the functionality).

During each phase, in order to engage and encourage users to continue their collaboration, IMS allows the collaborative resolution of problems, emerged during the implementation of the idea. Moreover, IMS transparently associates additional information to each phase as follows:

- Update of the status of implementation of the idea

- Resources (technical, human, ...) associated with implementation of the ideas

- Information about any problems encountered in the implementation phase

- Financial data

- Timelines 
Execution and Monitoring. The final stage of the process of co-creation is to run the service and continuously monitor the results. The monitoring phase is very important because it allows evaluating and monitoring the success or failure of the new service through the feedback received from users and the PA.

Monitoring techniques are questionnaires, interviews, surveys, reviews, and the feedback, collected by the Idea Management System. Also in this phase sentiment analysis tools are used. The functionality of "Analysis, filtering, and tracking of ideas" provides statistics and graphs that depict the performance of the Idea Management System over time. All contents of the system, in the form of a summary table and the frequency of interactions within the community, can be displayed.

The actors involved are: PA, citizens, and community of users. The feedback of the users and the data collected allow generating suggestions for improvement and new ideas that will reopen the cycle.

\section{Case Study "Lecce 2019 - IMS"}

The first four steps of the proposed framework were tested in the city of Lecce on the occasion of the initiative for Lecce candidacy as European Capital of Culture 2019.

Counting 90,000 citizens, Lecce is a mid-sized city, which represents the most important province of the Salento region, located in the "heel" of the Italian "boot". Even though Lecce is known for its great cultural, artistic, and naturalistic heritage, it can also be considered as a typical example of southern Italian city from a socio-economic point of view: poor in infrastructure, with high and increasing unemployment rates. However, despite this disadvantageous context, remarkable investments in university research and tourism sectors have been taking place over the last few years, making Lecce an area of attraction also at international level.

The Municipality of Lecce (Italy) has decided to change the approach for the creation of a shared path towards a social model, in which the direct participation and collaboration of the citizens is included in order to generate innovation.

Public administration and citizens are generally not coordinated with each other, since the traditional approach of urban planning is top-down and often does not meet citizens' needs. In the guidelines of the Bid Book ${ }^{5}$ for the candidacy of Lecce as European Capital of Culture 2019, one of the main criteria of the evaluation is "the city and citizens", referring to concrete initiatives that must be launched to attract local neighboring and foreign citizens' interest and participation.

Citizens' involvement and their needs definition are important elements for Lecce. For these reasons the Municipality of Lecce organized LUAC's (urban, open, creative laboratories): a kind of informal debate aiming to satisfy citizens' participation. "Lecce 2019 - Idea Management System" was adopted to integrate LUAC's and other initiatives that enable interaction between citizens.

As regards the access to the platform, the correlation between the number of visits and the interest shown by citizens and local associations towards the initiative Lecce 2019 is evident. The launch of the website, which took place in July 2013, was accompanied by a steady increase with a peak in September, close to the deadline for the submission of the bid book. Thereafter there was a decline in November after the announcement of the results of the first phase of selection; this shows that the number of accesses and interactions is strongly influenced by the diffusion of the various initiatives and different maturities (see Table 1).

\footnotetext{
${ }^{5}$ Lecce 2019 (2013) Reinventing Eutopia, Application for the title of European Capital of Culture, September 2013 available at http://www.lecce2019.it/2019/bidbook.php
} 
Table 1. Detailed data of the idea collection process

\begin{tabular}{|l|l|l|l|}
\hline \multicolumn{2}{|c|}{ Time } & \multicolumn{2}{c|}{ Issues } \\
\hline Time since first idea posted & 1 years 6 months & Number of idea & 2248 \\
\hline Time since last idea posted & 1 months & Number of idea contests & 9 \\
\hline Time since last contest created & 1 years 6 months & Total users & 1226 \\
\hline Time since last comment posted & 1 months & Total votes cast & 328 \\
\hline
\end{tabular}

In this regard, Caritas Diocesana of Lecce proposed, within the IMS, the idea of creating a network of solidarity aimed at collecting and distributing food. This idea has been voted and commented by other voluntary associations (Red Cross of Lecce, Comunità Emmanuel, etc.) and by some local shops, all enthusiastic and ready to participate.

The Municipality of Lecce by considering the idea interesting for the local community and evaluating, thanks to sentiment analysis indicators, the interest shown on the web for this topic, has intervened, proposing itself as guarantor and coordinator of this network. Meetings and focus groups were organized in order to create a "network of solidarity" involving several actors, such as: voluntary associations, Confcommercio, Confesercenti, Confindustria, and the managers of the Puglia@service project. The latter have given their availability to implement a web/mobile application able to facilitate the matching of demand and supply of unsold food. The execution and monitoring phase is in progress.

\subsection{Idea Management System's Main Functions}

Our research on IMS has mainly focused on three macro-categories in order to select the best solution for public administration usage.

1. Solution derives from the European research ${ }^{6}$

2. Solution includes market tools of $\mathrm{IMS}^{7}$

3. Solution includes Open Source tools ${ }^{8}$

Having investigated all the aforementioned categories' solutions, the implementation of the "Lecce 2019 - IMS" was performed by using the tool Gi2MO IdeaStream ${ }^{9}$. It consists of a set of modules able to customize Drupal [27] in order to implement it as a system of Idea Management.

The decision to use Open Source (OS) software offers the following benefits:

- Cost-effectiveness: Since the cost of proprietary software is a considerable expense, the use of OS software gives the possibility to switch to other suppliers to receive support.

- Security and reliability: The software is more secure because the user can view the source code and improve it. The product is, therefore, more stable and always updated.

- Freedom: OS software allows the interaction between multiple systems in a simple and fast way having the source code always available.

The implemented Idea Management functions are based on two fundamental entities: Contest and Ideas:

- Contests represent the area of interest requiring new solutions and approaches to improve the conditions of the public goods ${ }^{10}$.

\footnotetext{
${ }^{6}$ In particular three projects co-funded by the European Commission have been deepen: COIN (COIN Project, 2008), LABORANOVA (LABORANOVA 2006), ECOLEAD (ECOLEAD, 2008)

${ }^{7}$ Tools such as Accept360 Idea Management, CREAX Innovation Suite 3.1, CRITflow, DataStation, e-tipi, Hype Innovation, IBM Idea Factory, IDEAYST IDEALYST, 2014), INPAQT, ID8systems, Idea Management Platform 2.0

${ }^{8}$ Open source tool such as IdeaTorrent, BBYIDX, id-Force, OpenideaL - Idea Management, Gi2MO, Idea Box

${ }^{9}$ GI2MO ideastream (2014). http://www.gi2mo.org/.
} 
- Ideas represent the possible solutions proposed to the satisfaction of the needs expressed by the Contests.

These two entities will be the prerogative of two types of users. On the one hand, the Contest can be expressed by people representing Public Administration; while, on the other hand, the ideas can be expressed freely by the citizens.

The two categories (Contest and Ideas), despite being conceptually different, are characterized by several common elements: they can be represented by textual description and the title, they both can have as attachments some documents and files, they can belong to categories that allow the best definition of their scope and context, they can have some associated tags, they can be shared on social networks, and, finally, the contests and ideas can be defined by only one author.

In addition to these common elements, Contests and Ideas are characterized by some distinctive features.

In particular, the category Contests has a starting date, from which the contest will be visible and available to the community, and an end date or closure, from which nobody can contribute with the formulation of new ideas. The Ideas have some unique characteristics: the ability for the main author and co-authors to edit the content of the idea, still preventing the co-authors to add or remove other collaborators (this functionality remains the prerogative of the author of the idea).

As already mentioned earlier, in order to better manage the proposed ideas by the community, IMS provides the idea life-cycle management, and, to realize it, the management team, called "board", is provided. This team is composed of the idea/contest author and also by a group of selected users.

The main functionalities that IMS offers to its users can be divided into four categories, namely, systems administration tool, co-creation tools for public administration, co-creation tools for citizen users, and information tools for citizen users (see Table 2).

Table 2. IMS main functionalities

\begin{tabular}{|c|c|}
\hline 1. System administration tool & 2. Co-Creation tools for public administration \\
\hline User Administration & Managing an idea contest \\
\hline Role administration & Selecting and promoting an idea \\
\hline Association members-Role Administration & Rate/Comment/Share an idea or idea contest \\
\hline 3. Co-creation tools for citizen users & 4. Information tools for Citizen user \\
\hline Idea management & \multirow[t]{3}{*}{ Share / View ideas and idea contest } \\
\hline Idea co-worker Management (add/remove collaborators) & \\
\hline Rate/Comment/Share an idea or idea contest & \\
\hline
\end{tabular}

\subsection{Sentiment Analysis Case Study}

To test the effectiveness of the Sentiment Analysis tool, developed to support the Idea Management System, we have considered the tweets about the event Lecce 2019, and we have analyzed the sentiment. We collected a corpus of tweets using the Twitter search API between September 2, 2014 and November 17, 2014, the period in which there were relatively many twitter messages about the event. We extracted tweets using query-based search to collect the tweets relating to "Lecce2019" and "noisiamolecce2019", hashtags most used for this topic. The

${ }^{10}$ Public goods includes fresh air, knowledge, lighthouses, national security, flood control system, street lighting,etc. (http://en.wikipedia.org/wiki/Public_good) 
resulting dataset contains 5000 tweets of which 3560 are re-tweets. Duplicates were automatically removed leaving a set of 2000 tweets with a class distribution as shown in Table 3 .

Table 3. Class distribution

\begin{tabular}{|l|l|l|}
\hline Class of the tweet/label & \multicolumn{1}{|c|}{ Number of tweets } & Percentage of tweets \\
\hline Positive & 455 & $23 \%$ \\
\hline Neutral & 1463 & $73 \%$ \\
\hline Negative & 82 & $4 \%$ \\
\hline Total & 2000 & \\
\hline
\end{tabular}

In order to achieve a training set for creating a language model useful to the sentiment classification, a step of manual annotation was performed using the following 3 labels:

- Positive: Tweets that carry positive sentiment towards the topic Lecce 2019.

- Neutral: Tweets that do not carry any sentiment towards the topic Lecce 2019 or Tweets which do not have any mention or relation to the topic.

- Negative: Tweets that carry negative sentiment towards the topic Lecce 2019.

Before performing the classification of sentiment, the text has been processed by the preprocessing component. Seven classification loops were performed, each with different approaches for the features selection, and different fixed training dimensions. An accuracy of $77.6 \%$ was achieved using an individual sentiment classification algorithms and unigrams features with the stop-words and repeated letters elimination [28].

\section{Literature Review}

\subsection{Idea Management System}

Literature on Idea Management (IM) are predominantly associated with innovation management in organizations [29]. As reported by Baumgartner, the practices on innovation management are not new and have been introduced in several organizations much before the IT systems explosion (e.g., 30-years history of innovation management in Toyota had been always oriented on the road to the capture of novel ideas) [30]. However, what nowadays is known as 'idea management' in IT sector, has been created in reference to systems that appeared in the late 90s [31]. Idea Management Systems are the tools for collecting community's ideas for innovation purposes. In order to evaluate captured ideas precisely, Westerski et.al [10] have tried to resolve the problem by introducing annotation of ideas, through which the characteristics of ideas can be described highlighting their distinctive features.

Reviewing IT related literature remarks the development of IM dealing with applications of IMS. Xie and Zhang, for instance, have designed an IMS to support the process of idea generation, evaluation, improvement, and implementation [32]. The work of Westerski et al. [33] deals with the development of IMS and extends it from being nothing more than a box where employees could submit their ideas on a piece of paper to the web 2.0 techniques. Such transformation allows complex submission of data and data handling in IMS. They also suggests the use of semantic web principles to link organizational systems for better idea assessments [34]. IMS can also be considered as a sharing point among users and organizations [35], besides, in this manner it can be utilized as a managing and controlling tool for open innovation [36]. An example of Idea Management System is OpenIDEO ${ }^{11}$ that enables people to collaborate in developing innovative solutions to press social and environmental challenges. OpenIDEO is

\footnotetext{
${ }^{11} \mathrm{https} / / /$ openideo.com/about-us
} 
based on a collaborative process characterized by six steps. These tools, already adopted within enterprises, are able to avoid failures due to the implementation of products or services that do not suit market needs.

Idea Management System can be defined, therefore, as a process of needs recognition and ideas generation and evaluation [7], [37]. Those platforms aim to help all aforementioned practices of idea management and allow organizations to collect community ideas during enterprise procedures [38]. The main contribution of this paper is to develop an approach, based on idea life-cycle, which uses the concept of open innovation. We apply the proposed approach in the context of Public Administration in order to co-create innovative public services. In this approach, all steps of life-cycle are supported by the Idea Management System that interacts through a number of technological and methodological tools to facilitate collaboration and cocreation.

\subsection{Sentiment Analysis}

Sentiment Analysis (SA) and Opinion Mining study, analyze, and classify documents of the opinions expressed by the people about a product, service, event, organization, or a person. The objective of this area is the development of linguistic analysis methods that allows identifying the polarity of the opinions.

In the last decade, SA had a strong development, thanks to the large presence in the World Wide Web of an increasing number of documents generated by users and thanks to diffusion of social network.

In 2001 the paper of Das [39] and Tong [40] began to use the term "sentiment" refering to the automatic analysis of evaluative text and tracking of the predictive judgments. Since that time, the awareness of the research problems and the opportunity related to SA and Opinion Mining has been growing. The growing interest in SA and Opinion Mining is partly due to the different application areas: in commercial field - to the analysis of the review of products [41]; in political field - to identify the electorate mood and therefore the trend in the voting (or abstaining) [42]; etc.

In social environments, the SA can be used as a survey tool that allows to understand the existing points of view: for example, to understand the opinion that some people have about a subject, to predict the impact of a future event, or to analyze the influence of a past event on a recent one [43].

The big data technologies, the observation methods, and the analysis of the behavior on the web, make SA an important decision making tool for the analysis of social network, able to develop relation, culture, and sociological debate.

The SA carried out in social networks allows public administrations to identify and meet user's needs and also enables citizens to affect the service delivery and to participate in the creation of a new services, or even to identify innovative uses of existing services [44].

\section{Results and Future Developments}

The proposed approach is used in the context of Public Private Partnership for a charitable cause. This need was expressed by citizens through the IMS platform and has been taken into consideration by the Local Government. The idea was to create a 'food bank' for collecting the excess food from restaurants and supermarkets and distribute it among the needy. Based on this idea, a specific platform, which enables both donators and poor citizens to interact, has been developed. Such system reduces the food waste and, at the same time, increases the support for needy citizens. It is one of the significant preliminary results of the implemented system, which has been achieved through the exploitation of the platform of IMS. 
IMS tool could be adapted to different contexts and to a variety of interactions between government and citizens, but it requires further improvements. A more user-friendly interface and a mobile version could be valuable additions. The moderator can be in charge of preserving the platform essence (aggregator of ideas and perspectives), since he/she could have alternatively two different roles: spamming activities checking and inappropriate content detection. However, it is crucial to pay attention to the second role since it could even be seen as an attempt to restrain freedom, which is one of the fundamental characteristic of the IMS tool.

Another lesson learned, during the test of IMS, concerns its adoption by citizens. The outcome of the statistical analysis has shown that the use of "Lecce 2019 - IMS" was closely correlated to the candidacy of Lecce as European Capital of Culture 2019. In the future, the IMS will be implemented in order to stimulate its daily usage as a social innovation tool.

A new extension, called "Social sentiment index" is currently under development. This new extension aims at integrating the potential of sentiment analysis to identify the greatest interest of the community. However, the usage of an Idea Management System to support strategic planning in an open environment, such as urban areas, introduces a problem: administrators need further tools to prioritize efficiently interventions in the urban context. For this reason, we are working to extend the capabilities of the Idea Management System by introducing an algorithm that could calculate the user participation. The Social sentiment index will be calculated from a set of input parameters, resulting not only from the Idea Management System, but also by means of the major social networks like Facebook, Twitter, Google+, and LinkedIn. On the other hand, sentiment analysis tools, by using specific algorithms as well as semantic function, will have the purpose to simplify and to categorize the content. Founded on the concept of interoperability, the project proposes a number of solutions using metadata and providing new methods of evaluation: metrics based on opinion mining, taxonomy, and categorization of innovation, as well as metrics based on reports of the idea.

\section{References}

[1] V. Ramaswamy, "Co-Creation of Value - Towards an Expanded Paradigm of Value Creation," Marketing Review St. Gallen, vol. 26(6), pp. 11-17, 2009. Available: http://dx.doi.org/10.1007/s11621-009-0085-7

[2] D. Boyle, M. Harris, "The Challenge of Co-production," 2009.

[3] T. O’Reilly, “What Is Web 2.0,” O’Reilly Media, Inc., 2006.

[4] G. Mulgan , B. Sanders, R. Ali, S. Tucker, "Social Innovation what it is, why it matters and how it can be accelerated," 2007.

[5] A. H. Van de Ven, M. S. Poole, "Methods for Studying Innovation Development in the Minnesota," Organ. Sci., vol. 1(3), pp. 313-335, 1990. Available: http://dx.doi.org/10.1287/orsc.1.3.313

[6] A. J. Berkhout, D. Hartmann, P. Van Der Duin, R. Ortt, "Innovating the innovation process," Int. J. Technol. Manag., vol. 34, no 3/4, pp. 390-404, 2006. Available: http://dx.doi.org/10.1504/IJTM.2006.009466

[7] B. Vabdenbosch, A. Saatcioglu, S. Fay, "Idea Management: A Systemic View,” J. Manag. Stud., vol. 43, no. 2, pp. 259-288, 2006. Available: http://dx.doi.org/10.1111/j.1467-6486.2006.00590.x

[8] H. W. Chesbrough, “The Era of Open Innovation,” Manag. Innov. Chang., vol. 127, pp. 34-41, 2006.

[9] H. W. Chesbrough, "Open Innovation: The New Imperative for Creating and Profiting from Technology," Harvard Business Press, 2006.

[10] H. W. Chesbrough, W. Vanhaverbeke, J. West, “Open Innovation: Researching a New Paradigm,” Oxford University Press, 2006.

[11] C. E. Seidel, B. E. P. Thapa, R. Plattfaut, B. Niehaves, "Selective Crowdsourcing for Open Process Innovation in the Public Sector - Are Expert Citizens Really Willing to Participate?" in Proc. of the 7th International Conference on Theory and Practice of Electronic Governance, ACM, New York, NY, USA, pp. 64-72, 2013. Available: http://dx.doi.org/10.1145/2591888.2591899 
[12] N. Edelmann, J. Höchtl, M. Sachs, "Collaboration for Open Innovation Processes in Public Administrations," In: Y. Charalabidis, S. Koussouris (eds.) Empowering Open and Collaborative Governance, Springer Berlin Heidelberg, pp. 21-37, 2012. Available: http://dx.doi.org/10.1007/978-3-642-27219-6_2

[13] A. Collm, K. Schedler, "Managing Crowd Innovation in Public Administration," Int. Public. Manag. Rev., vol. 13, no. 2, pp. 1-18, 2012.

[14] D. Hilgers, C. Ihl, "Citizensourcing: Applying the concept of open innovation to the public sector," Int. J. Public. Particip., vol. 4, no.1, pp. 67-88, 2010.

[15] G. Koch, J. Füller, S. Brunswicker, "Online crowdsourcing in the public sector: How to design open government platforms,” Online Communities and Social Computing, vol. 6778, LNCS, pp. 203-212, 2011. Available: http://dx.doi.org/10.1007/978-3-642-21796-8_22

[16] B. J. Jansen, M. Zhang, K. Sobel, A. Chowdury, “Twitter power: Tweets as electronic word of mouth,” J. Am. Soc. Inf. Sci. Technol., vol. 60(11), pp. 2169-2188, 2009. Available: http://dx.doi.org/10.1002/asi.21149

[17] B. O'Connor, R. Balasubramanyan, B. R. Routledge, N. A. Smith, "From tweets to polls: Linking text sentiment to public opinion time series," In Proc. of the Fourth International Conference on Weblogs and Social Media, ICWSM '10, Washington, DC, USA, 2010.

[18] A. Tumasjan, T. O. Sprenger, P. G. Sandner, I. M. Welpe, "Predicting elections with Twitter: What 140 characters reveal about political sentiment," in Proc. of the Fourth International Conference on Weblogs and Social Media, ICWSM '10, Washington, DC, USA, pp. 178-185, 2010.

[19] E. Kouloumpis, T. Wilson, J. Moore, “Twitter sentiment analysis: The good the bad and the OMG!” in Proc. of the Fifth International Conference on Weblogs and Social Media, ICWSM '11, Barcelona, Catalonia, Spain, 2011 .

[20] M. Salathe., S. Khandelwal, “Assessing Vaccination Sentiments with Online Social Media: Implications for Infectious Disease Dynamics and Control," PLoS Computational Biology, vol. 7(10), 2011. Available: http://dx.doi.org/10.1371/journal.pcbi.1002199

[21] B. Mandel, A. Culotta, J. Boulahanis, D. Stark, B. Lewis, J. Rodrigue, “A Demographic Analysis of Online Sentiment during Hurricane Irene," in Proc. of the Second Workshop on Language in Social Media, LSM 12, Stroudsburg, pp. 27-36, 2012.

[22] J.-M. Xu, K.-S. Jun, X. Zhu, A. Bellmore, "Learning from bullying traces in social media," in Proc. of the 2012 Conference of the North American Chapter of the Association for Computational Linguistics: Human Language Technologies, pp. 656-666, 2012.

[23] S. Asur, B. A. Huberman, "Predicting the future with social media," in Proc. of the 2010 International Conference on $132 \mathrm{Web}$ Intelligence and Intelligent Agent Technology, WI-IAT '10, Washington, DC, USA. IEEE Computer Society, vol. 1, pp. 492-499, 2010. Available: http://dx.doi.org/10.1109/wi-iat.2010.63

[24] A. Bakliwal, J. Foster, J. van der Puil, R. O'Brien, L. Tounsi, M. Hughes, "Sentiment Analysis of Political Tweets: Towards an Accurate Classifier," in Proc. of the Workshop on Language in Social Media (LASM 2013), Atlanta, Georgia, pp. 49-58, 2013.

[25] A. Westerski, C. A. Iglesias, F. T. Rico, "A Model for Integration and Interlinking of Idea Management Systems," Metadata and Semantic Research,. Springer Berlin Heidelberg, vol. 108 of the series Communications in Computer and Information Science, pp. 183-194, 2010. Available: http://dx.doi.org/10.1007/978-3-642-16552-8_18

[26] V. Allee, "A Value Network Approach for Modeling and Measuring Intangibles," Transparent Enterp., Madrid, 2002.

[27] S. Corlosquet, R. Delbru, T. Clark, S. Decker, "Produce and Consume Linked Data with Drupal!" Semantic Web - ISWC 2009. Springer Berlin Heidelberg, vol. 5823, LNCS, pp. 763-778, 2009. Available: http://dx.doi.org/10.1007/978-3-642-04930-9_48

[28] A. Corallo, L. Fortunato, M. Matera, M. Alessi, A. Camillò, V. Chetta, E. Giangreco, D. Storelli, "Sentiment Analysis for Government: an optimized approach," Machine Learning and Data Mining in Pattern Recognition, vol. 9166, LNCS, pp. 98-112, 2015. Available: http://dx.doi.org/10.1007/978-3-319-21024-7_7

[29] A. R. V. Jensen, “A literature review of idea management,” in Proc. 9th Nord. Conf. 22-24 Aalborg Univ. Denmark, 2012.

[30] J. P. Baumgartner, “Big and little innovation,” Report 103. 27, 2004. 
[31] C. Rozwell, et al., Survey of Innovative Management Technology. Gart Res (Research Note: No. M-15-1388), 2002.

[32] L. Xie, P. Zhang, ”Idea Management System for Team Creation,” J. Softw., vol. 5, no. 11, pp. 1187-1194, 2010. Available: http://dx.doi.org/10.4304/jsw.5.11.1187-1194

[33] A. Westerski, C. A. Iglesias, T. Nagle, "The road from community ideas to organisational innovation," Int. J. Web Based Communities, vol. 7, no. 4, pp. 493-506, 2011. Available: http://dx.doi.org/10.1504/IJWBC.2011.042993

[34] E. Giangreco, L. Marasso, V. Chetta, L. Fortunato, C. Perlangeli, "Modeling tools of service value networks to support social innovation in a Smart City," Innovation and the Public Sector, vol. 21: Electronic Government and Electronic Participation. IOS Press Ebooks, pp. 206-215, 2014. Available: http://dx.doi.org/10.3233/978$1-61499-429-9-206$

[35] W.-F. Tung, S.-T. Yuan, J.-R. Tsai, “A custom collaboration service system for idea management of mobile phone design," Hum. Factors Econ. Manuf. Serv. Ind., vol. 19(5), pp. 495-509, 2009. Available: http://dx.doi.org/10.1002/hfm.20147

[36] E. Enkel, O. Gassmann, H. Chesbrough, “Open R\&D and open innovation: exploring the phenomenon,” R\&D Manag., vol. 34, no. 4, pp. 311-316, 2009. Available: http://dx.doi.org/10.1111/j.1467-9310.2009.00570.x

[37] L. Maraso, E. Giangreco, D. Storelli, V. Chetta, A. Camilo, "Idea Management System for Smart City Planning,” Interdiscip. Stud. J., vol. 3, pp. 227-236, 2014.

[38] A. Westerski, T. Dalamagas, C. A. Iglesias, "Classifying and comparing community innovation in Idea Management Systems," Decision Support Syst., vol. 54(3), pp. 1316-1326, 2013. Available: http://dx.doi.org/10.1016/j.dss.2012.12.004

[39] S. R. Das, M. Y. Chen, "Yahoo! for Amazon: Extracting market sentiment from stock message boards," in Proc. of the Asia Pacific Finance Association Annual Conference (APFA), 2001.

[40] R. M. Tong, “An operational system for detecting and tracking opinions in on-line discussion," in Proc. of the SIGIR Workshop on Operational Text Classification (OTC), 2001.

[41] K. Dave, S. Lawrence, D. M. Pennock, "Mining the peanut gallery: Opinion extraction and semantic classification of product reviews," in Proc. of the 12th International Conference on World Wide Web, pp. 519528, 2003. Available: http://dx.doi.org/10.1145/775152.775226

[42] F. Neri, C. Aliprandi, F. Camillo, "Mining the Web to monitor the Political Consensus," in Counterterrorism and Open Source Intelligence, Lecture Notes in Social Networks, part III, pp. 391-412, 2011. Available: http://dx.doi.org/10.1007/978-3-7091-0388-3_19

[43] A. Kale, A. Karandikar, P. Kolari, A. Java, T. Finin, A. Joshi, "Modeling trust and influence in the blogosphere using link polarity," in Proc. of the International Conference on Weblogs and Social Media (ICWSM), 2007.

[44] C. Dolicanin, E. Kajan, D. Randjelović, B. Stojanović, "Handbook of Research on Democratic Strategies and Citizen-Centered E-Government Services.” IGI Global, 2014. Available: http://dx.doi.org/10.4018/978-14666-7266-6 